\title{
Prognostic Factors in Advanced Non-Small-Cell Lung Cancer Patients: Patient Characteristics and Type of Chemotherapy
}

\author{
Salah Abbasi and Ahmed Badheeb \\ Clinical Oncology Department, King Hussein Cancer Center, Queen Rania Al-Abdullah Street, Al-Jubeiha, Amman 11941, Jordan \\ Correspondence should be addressed to Salah Abbasi, sabbasi@khcc.jo
}

Received 29 September 2010; Accepted 24 November 2010

Academic Editor: Kaoru Kubota

Copyright () 2011 S. Abbasi and A. Badheeb. This is an open access article distributed under the Creative Commons Attribution License, which permits unrestricted use, distribution, and reproduction in any medium, provided the original work is properly cited.

\begin{abstract}
Eleven prognostic factors were retrospectively analyzed in 270 newly diagnosed patients with advanced non-small-cell lung cancer including age, sex, performance status, histology, stage, smoking status, hemoglobin level, forced expiratory volume in one second (FEV1), weight loss $>5 \%$ in 3 months preceding therapy, number of involved organs, and type of first-line chemotherapy. Response rate was $35.6 \%$, and median survival was 8.2 months ( $95 \% \mathrm{CI}, 7.8$ to 8.7$)$ for the whole group. Age $\leq 60$ years $(P=.016)$, FEV1 $\geq 2 \mathrm{~L}$ $(P=.03)$, and the use of platinum/docetaxel $(P<.0001)$ were significantly associated with an improved survival. Histology did not affect outcome in the absence of targeted therapies.
\end{abstract}

\section{Introduction}

Lung cancer is the leading cause of cancer deaths among both men and women in the world. Non-small-cell lung cancer (NSCLC) represents 75-85\% of all lung cancer cases. Approximately, two-thirds of NSCLC patients have advanced stage at diagnosis beyond curative resection.[1] The median survival time for patients with untreated metastatic NSCLC is only 4-5 months, with a survival rate at one year of only $10 \%$ [2]. However, a meta-analysis has demonstrated that, as compared with best supportive care, chemotherapy results in a small improvement in survival in patients with advanced NSCLC [3]. Newer chemotherapy combinations showed a response rate of $19-32 \%$ and a median survival time of 7.9 to 11.3 months $[4,5]$. These numbers are also improved significantly with the use of targeted therapies although results have been variable among patients. This difference in outcome among patients with the same clinical stage of the disease suggests that advanced NSCLC is a heterogeneous disease. Additionally, some patients experience weight loss and some have a significant number of comorbidities. This wide spectrum of clinical features of patients with stage IIIB/IV NSCLC probably contributes to disparities in outcomes seen in different clinical trials. Although prognostic variables have been described for advanced stage NSCLC [6], no reports have addressed this issue in our patient population. Therefore, this study aims to determine the patient and tumor variables that are associated with improved outcome in Jordanian patients who are undergoing first-line palliative chemotherapy for stage IIIB/IV NSCLC. Also, we looked at the outcome of chemotherapy in our patients with advanced NSCLC, who in general have limited access to new targeted therapies and molecular testing.

\section{Patients and Methods}

We retrospectively reviewed the clinical records of 321 patients with a pathological diagnosis of advanced, noncurative stage IIIB or IV NSCLC, who were seen and treated at King Hussein Cancer Center (KHCC) in Jordan between 2007 and 2009. More than $80 \%$ of lung cancer patients in Jordan are treated at KHCC, which makes the sample very representative of the Jordanian population.

Tumors were classified according to the recommendations of the WHO. Tumor staging was done according to the international staging system for NSCLC 1997 [7]. All patients were staged by computed tomography scan of the chest, abdomen, and pelvis and bone scan. Positron emission tomography scan and brain imaging were used for staging in 
some patients upon the discretion of the treating physician if needed.

Data collected included patients gender, age at diagnosis, Eastern Cooperative Oncology Group performance status (PS), histology, stage, smoking status, history of weight loss more than $5 \%$ in 3 months preceding diagnosis, hemoglobin level $(\mathrm{Hb})$ at diagnosis (in $\mathrm{g} / \mathrm{dL}$ ), forced expiratory volume in one second (FEV1) at diagnosis, number of involved organs by metastasis, and type of first-line chemotherapy for treated patients.

A total of 270 patients out of the 321 were treated with first-line chemotherapy (according to treating physician discretion) and were included in the analysis for response rate, survival time, and prognostic variables. Fifty one patients were not treated either because of poor PS or patient's refusal and were not included in the analysis for survival or prognosis. Time to progression (TTP) was defined as the time from first day of chemotherapy to first documentation of progressive disease. Survival time was measured from the date of first day of chemotherapy to date of death from any cause or date of last patient contact.

This work has been approved by the institutional review board at KHCC.

\section{Statistical Analysis}

All treated $(n=270)$ patients were included in the statistical calculations. Duration of survival was assessed using the Kaplan-Meier method. Variables were studied for influence on survival in a univariate analysis by using the Log-rank test, and in a multivariate analysis using a stepwise Cox proportional hazards regression analysis. The results were considered significant at the 0.05 level. Statistical analysis was performed by using the SAS version 9.1 (SAS Institute Inc., Cary, NC).

\section{Results}

4.1. Patient's Characteristics. Among the 321 patients with advanced NSCLC, 273 (85\%) were males and 48 (15\%) were females with a male: female ratio of $5.7: 1$. Two hundreds and nine patients $(65 \%)$ were above the age of 60 years at diagnosis. The most common histology was adenocarcinoma (181, 56.4\%), while squamous cell carcinoma and large cell carcinoma accounted for $31 \%$ and $6.2 \%$, respectively.

Most patients were smokers at time of diagnosis (78.2\%), but only $21 \%$ of patients reported a history of significant weight loss. The majority of patients had metastasis to single organ $(215,67 \%)$, rather than two organs or more (106, $33 \%)$. The median time between first symptoms appearance and actual diagnosis of lung cancer was 4 months (range, 1-12).

4.2. Response and Outcome of Therapy. Characteristics of the 270 patients who received palliative chemotherapy are shown in Table 1 . The majority of patients received platinum/docetaxel combination $(183,67.8 \%)$, while the rest of patients received other therapies (platinum/gemcitabine

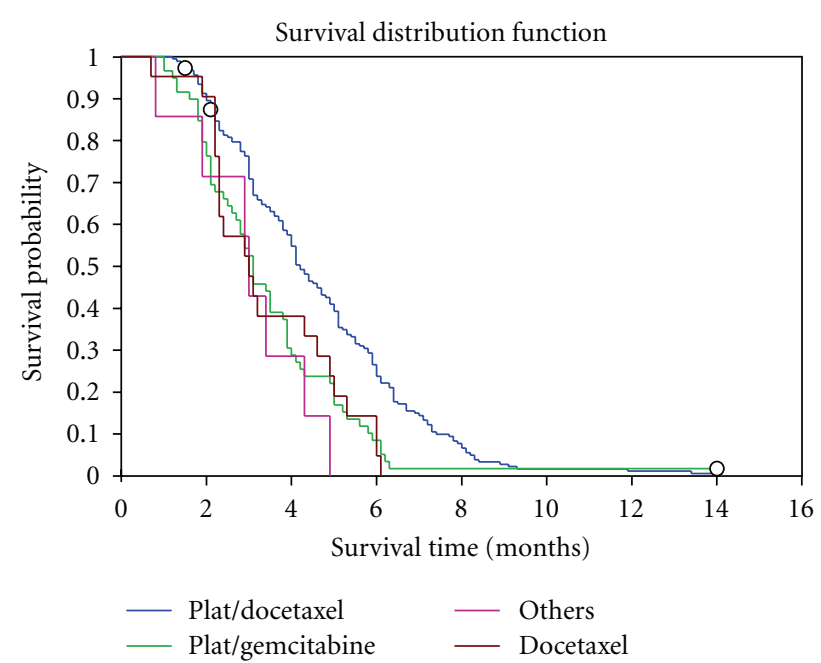

Figure 1: Kaplan-Meier estimate of time to disease progression among advanced NSCLC patients treated with different chemotherapy regimens.

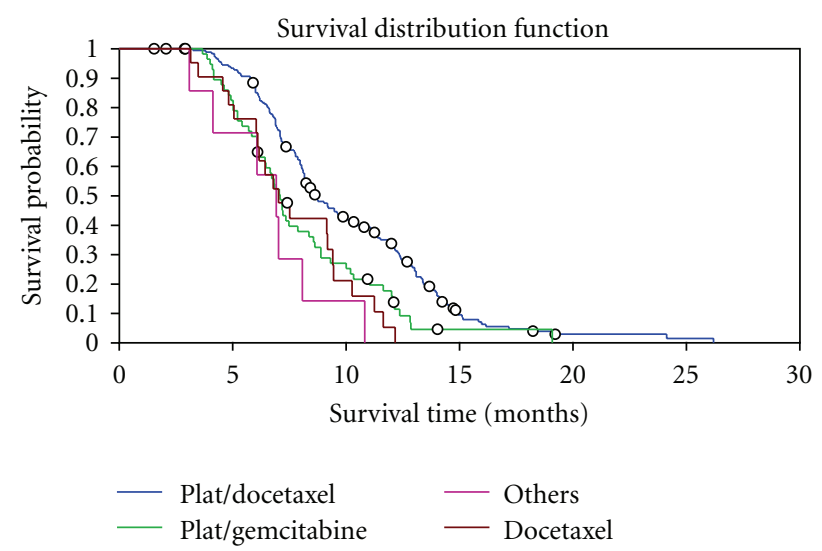

FIGURE 2: Kaplan-Meier estimate of overall survival among advanced NSCLC patients treated with different chemotherapy regimens.

in $21.9 \%$, other platinum-based doublets in $2.6 \%$, and single agent docetaxel in $7.8 \%$ ).

The overall response rate (ORR) (complete and partial remissions) for the whole group was 35.6\%, while $30 \%$ of patients achieved stable disease, for a disease control rate of $65.6 \%$. The ORR for platinum/docetaxel group was $38 \%(70 / 183)$ versus $30 \%$ for all other therapies combined $(26 / 87)$, and the difference was not statistically significant $(P=.3)$.

TTP was 3.9 months (95\% CI, 3.5-4.1) for the whole group of treated patients. TTP was significantly longer in the group of patients who received platinum/docetaxel $(4.2$ months) than for patients who received other therapies (3.1 months, $P<.0001$ ) as shown in Figure 1 .

The median survival time (MS) for the whole group of treated patients was 8.2 months (95\% CI, 7.8-8.7) with significantly higher MS for the platinum/docetaxel group over other therapies group ( 8.9 months versus 7.1 months, 
TABLE 1: Characteristics and univariate analysis of prognostic factors in treated NSCLC patients $(n=270)$.

\begin{tabular}{|c|c|c|c|}
\hline Characteristic & $\begin{array}{c}N \\
(\text { total }=270)\end{array}$ & $\begin{array}{c}\text { Median } \\
\text { survival } \\
\text { (months) }\end{array}$ & $\begin{array}{c}\text { Log-rank test } \\
P \text {-value }\end{array}$ \\
\hline \multicolumn{4}{|l|}{ Age (years) } \\
\hline$\leq 60$ & 108 & 8.6 & \multirow[b]{2}{*}{.016} \\
\hline$<60$ & 162 & 8.0 & \\
\hline \multicolumn{4}{|l|}{ Sex } \\
\hline Male & 228 & 8.2 & \multirow[b]{2}{*}{.59} \\
\hline Female & 42 & 8.3 & \\
\hline \multicolumn{4}{|l|}{ PS } \\
\hline 0 & 243 & 8.3 & \multirow{2}{*}{.25} \\
\hline $1+2$ & 27 & 6.7 & \\
\hline \multicolumn{4}{|l|}{ Histology } \\
\hline Adenocarcinoma & 152 & 8.4 & \multirow[b]{2}{*}{.31} \\
\hline Others & 118 & 8.1 & \\
\hline \multicolumn{4}{|l|}{ Stage } \\
\hline IIIB & 17 & 11.2 & \multirow[b]{2}{*}{.075} \\
\hline IV & 253 & 8.1 & \\
\hline \multicolumn{4}{|l|}{ Smoking status } \\
\hline Current & 209 & 8.1 & \multirow[b]{2}{*}{.79} \\
\hline Never/former & 61 & 8.59 & \\
\hline \multicolumn{4}{|l|}{$\mathrm{Hb}$} \\
\hline$\geq 12$ & 219 & 8.1 & \multirow[b]{2}{*}{.41} \\
\hline$<12$ & 51 & 10.2 & \\
\hline \multicolumn{4}{|l|}{ FEV1 } \\
\hline$\geq 2 \mathrm{~L}$ & 128 & 9.2 & \multirow[b]{2}{*}{.03} \\
\hline$<2 \mathrm{~L}$ & 113 & 8.0 & \\
\hline \multicolumn{4}{|l|}{ Weight loss } \\
\hline No & 190 & 8.2 & \multirow[b]{2}{*}{.89} \\
\hline Yes & 49 & 8.3 & \\
\hline \multicolumn{4}{|l|}{ Sites of metastasis } \\
\hline Single organ & 191 & 8.7 & \multirow[b]{2}{*}{.68} \\
\hline Two or more & 79 & 8.2 & \\
\hline \multicolumn{4}{|l|}{ Type of chemotherapy } \\
\hline Platinum/Docetaxel & 183 & 8.9 & \multirow{3}{*}{$<.0001$} \\
\hline Platinum based doublet & 66 & 7.1 & \\
\hline Docetaxel monotherapy & 21 & 7.0 & \\
\hline
\end{tabular}

Abbreviations: PS: performance status; Hb: hemoglobin level in g/dL; FEV1: forced expiratory volume in one second.

respectively, $P<.0001)$ as shown in Figure 2. Patients who achieved remission (partial or complete) had significantly higher MS than patients with stable disease or progressive disease (12.4 months versus 6 months, respectively, $P<$ $.0001)$.

4.3. Prognostic Variables. Univariate analysis of prognostic factors was performed on all treated patients with survival data (Table 1). In this analysis, the only statistically significant factors observed were age at diagnosis of 60 years or younger $(P=.016)$, FEV1 at diagnosis of 2 liters or more $(P=.03)$, and the use of platinum/docetaxel as first line chemotherapy $(P<.0001)$. Differences in sex, PS, histology, stage, smoking status, Hb level, weight loss, or sites of metastasis were not significantly predictive of survival outcome.

The results of Cox regression analysis demonstrated the association of FEV1 $\geq 2 \mathrm{~L}$ (The hazard ratio was $0.74(95 \%$ CI, 0.55-0.92), $P=.015$ ), age $\leq 60$ years (The hazard ratio was 0.61 (95\% CI, 0.45-0.84), $P=.002)$, and the use of platinum/docetaxel regimen (The hazard ratio was 0.48 (95\% CI, 0.28-0.80), $P=.0003$ ) as independent prognostic factors for survival. Survival was not influenced by other prognostic variables.

\section{Discussion}

The prognosis of patients with stage IV NSCLC is still poor, most large phase III trials have shown a median survival time from 8 to 10 months [8]. In our study, we achieved an ORR of $35.6 \%$, TTP of 3.9 months, and a median survival time of 8.2 months, consistent with international data. As expected, our outcome is lower than the median survival achieved with the use of new drugs and targeted therapies, which unfortunately are not widely available in Jordan and many other developing countries. This fact, beside the lack of molecular testing and the ability to do more costly and sophisticated genetic testing in advanced NSCLC patients, made it very important to us to look at clinical and pathological prognostic variables, which may help us in treating our patients and to better allocate our resources.

Albain et al. demonstrated that a good PS, female sex, age $\leq 70$ years, $\mathrm{Hb}$ level $>11 \mathrm{~g} / \mathrm{dL}$, and normal lactate dehydrogenase levels were associated with improved outcomes in patients with advanced NSCLC [9]. It is noteworthy that our study confirmed the prognostic significance of age on survival although we used a lower cutoff age (60 years, instead of 70 years) because of the lower life span of our population and earlier presentation with lung cancer (median age at presentation in this study was 62 years). One of the most important factors across many trials is PS. Patients with stage IV NSCLC who are compromised by their disease have poor survival compared to patients who are less compromised $[10,11]$. Our study did not show this value for PS most probably because our patients with PS 1 or 2 did not receive treatment (small number of patients).

Current smoking during treatment for small-cell lung cancer has been shown to impact negatively on survival [12]. This present study did not find a survival advantage with respect to sex or smoking status.

Pretreatment weight loss is generally regarded as a negative prognostic factor, but not all trials have reliably shown this $[13,14]$. Our study showed that weight loss more than $5 \%$ in the last 3 months preceding the diagnosis of NSCLC is not a significant prognostic variable.

FEV1 more than 2L was found to be associated with improved survival in patients with locally advanced NSCLC who undergo curative concurrent chemoradiation 
therapy, [6] which is consistent with our results in stage IV patients who undergo palliative chemotherapy alone.

Although our study was not designed to compare different chemotherapy regimens, it clearly showed that the use of platinum/docetaxel combination is superior to other regimens used here, and it is an independent risk factor for better TTP and MS, regardless of histology. This is especially important for our population, where new therapies like pemetrexed, bevacizumab, and other targeted therapies are costly and not readily available. Histology subtype does not reliably provide prognostic importance in patients with advanced NSCLC, especially in the absence of targeted therapies.

We hope that overall prognostic assessment and treatment decisions can be individualized by taking into consideration specific patient characteristics that have been shown to affect survival. Additionally, these prognostic factors can be used in future study designs to properly stratify patients.

\section{Conclusion}

Although our study is retrospective, includes small number of patients from single institution, and does not include molecular or genetic prognostic factors, it suggests that FEV1 $\geq 2 \mathrm{~L}$, age $\leq 60$, and the use of platinum/docetaxel as firstline chemotherapy in stage IIIB/IV NSCLC can be useful in predicting for more favorable outcome. Histology of NSCLC does not affect outcome in the absence of targeted therapies. Future studies should look at more specific molecular and genetic factors. One study in Jordan is ongoing now to look at the prevalence of epidermal growth factor receptor mutations rate and its association with clinical response and survival in response to Erlotinib in the Jordanian population.

\section{Conflict of interests}

None to be declared.

\section{References}

[1] A. Jemal, R. Siegel, E. Ward, Y. Hao, J. Xu, and M. J. Thun, "Cancer statistics, 2009," CA: Cancer Journal for Clinicians, vol. 59, no. 4, pp. 225-249, 2009.

[2] E. Rapp, J. L. Pater, A. Willan et al., "Chemotherapy can prolong survival in patients with advanced non-small-cell lung cancer-report of a Canadian multicenter randomized trial," Journal of Clinical Oncology, vol. 6, no. 4, pp. 633-641, 1988.

[3] Non-Small Cell Lung Cancer Collaborative Group, "Chemotherapy in non-small cell lung cancer: a metaanalysis using updated data on individual patients from 52 randomised clinical trials," British Medical Journal, vol. 311, no. 7010, pp. 899-909, 1995.

[4] J. H. Schiller, D. Harrington, C. P. Belani et al., "Comparison of four chemotherapy regimens for advanced non-small-cell lung cancer," New England Journal of Medicine, vol. 346, no. 2, pp. 92-98, 2002.

[5] F. Fossella, J. R. Pereira, J. Von Pawel et al., "Randomized, multinational, phase III study of docetaxel plus platinum combinations versus vinorelbine plus cisplatin for advanced non-small-cell lung cancer: the TAX 326 study group," Journal of Clinical Oncology, vol. 21, no. 16, pp. 3016-3024, 2003.

[6] F. O. Ademuyiwa, C. S. Johnson, A. S. White et al., "Prognostic factors in stage III non-small-cell lung cancer," Clinical Lung Cancer, vol. 8, no. 8, pp. 478-482, 2007.

[7] L. H. Sobin, C. Wittekind, and International Union Against Cancer (UICC), TNM Classification of Malignant Tumors, Wiley-Liss, New York, NY, USA, 6th edition, 2002.

[8] M. A. Socinski, D. E. Morris, G. A. Masters, and R. Lilenbaum, "Chemotherapeutic management of stage IV non-small cell lung cancer," Chest, vol. 123, supplement 1, pp. 226S-243S, 2003.

[9] K. S. Albain, J. J. Crowley, M. LeBlanc, and R. B. Livingston, "Survival determinants in extensive-stage non-small-cell lung cancer: the Southwest Oncology Group experience," Journal of Clinical Oncology, vol. 9, no. 9, pp. 1618-1626, 1991.

[10] K. E. Stanley, "Prognostic factors for survival in patients with inoperable lung cancer," Journal of the National Cancer Institute, vol. 65, no. 1, pp. 25-32, 1980.

[11] D. M. Finkelstein, D. S. Ettinger, and J. C. Ruckdeschel, "Longterm survivors in metastatic non-small-cell lung cancer: an Eastern Cooperative Oncology Group study," Journal of Clinical Oncology, vol. 4, no. 5, pp. 702-709, 1986.

[12] G. M. M. Videtic, L. W. Stitt, A. R. Dar et al., "Continued cigarette smoking by patients receiving concurrent chemoradiotherapy for limited-stage small-cell lung cancer is associated with decreased survival," Journal of Clinical Oncology, vol. 21, no. 8, pp. 1544-1549, 2003.

[13] P. D. Bonomi, D. M. Finkelstein, J. C. Ruckdeschel et al., "Combination chemotherapy versus single agents followed by combination chemotherapy in stage IV non-small-cell lung cancer: a study of the Eastern Cooperative Oncology Group," Journal of Clinical Oncology, vol. 7, no. 11, pp. 1602-1613, 1989.

[14] J. Klastersky, J. P. Sculier, G. Bureau et al., "Cisplatin versus cisplatin plus etoposide in the treatment of advanced nonsmall-cell lung cancer," Journal of Clinical Oncology, vol. 7, no. 8, pp. 1087-1092, 1989. 


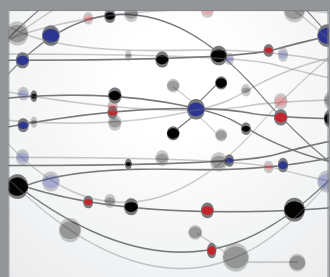

The Scientific World Journal
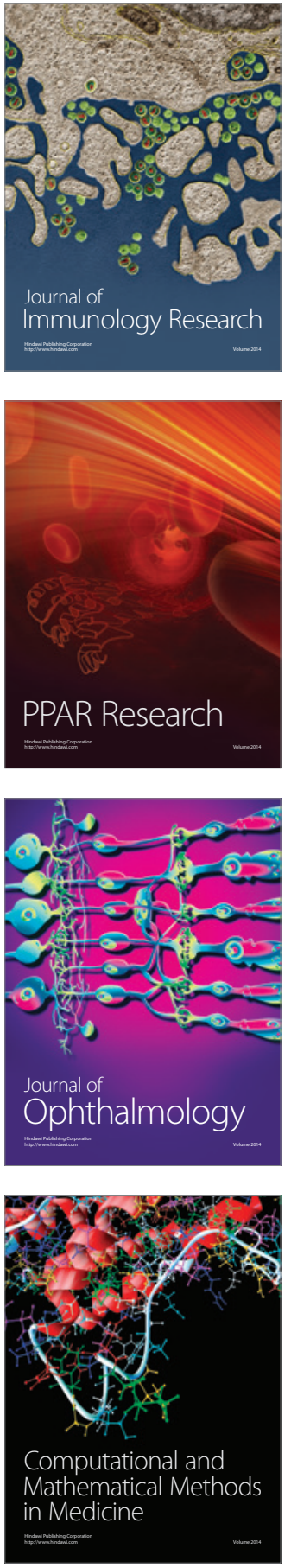

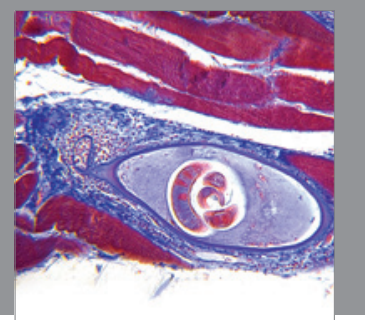

Gastroenterology

Research and Practice
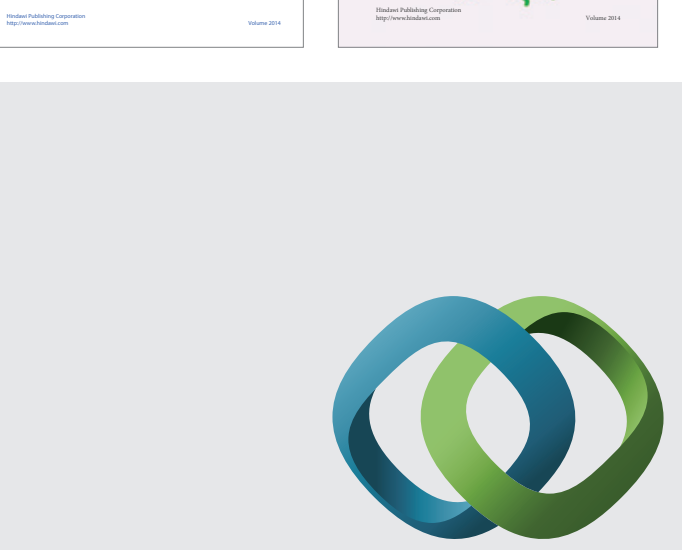

\section{Hindawi}

Submit your manuscripts at

http://www.hindawi.com
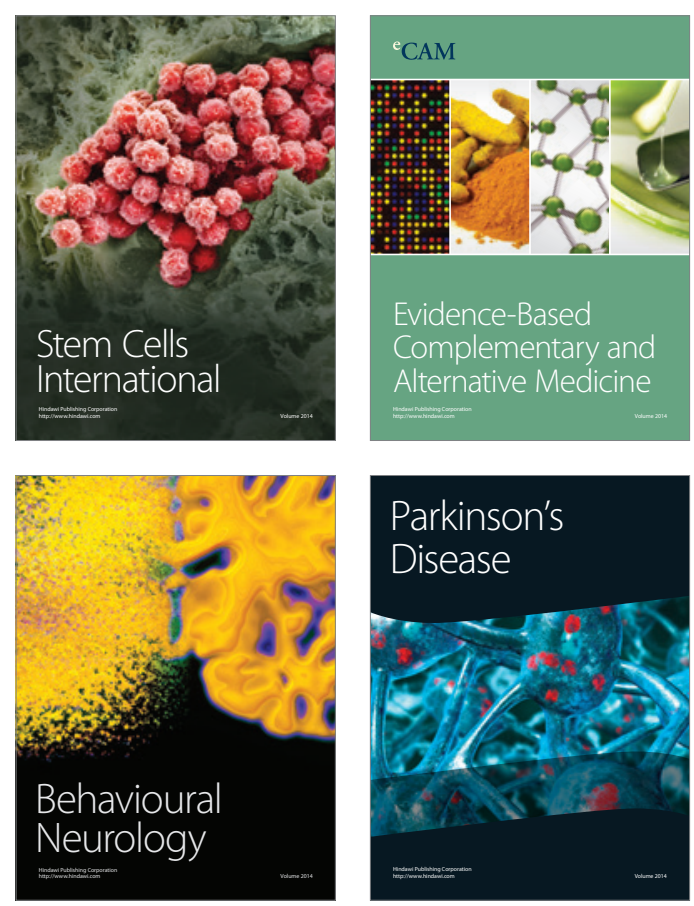

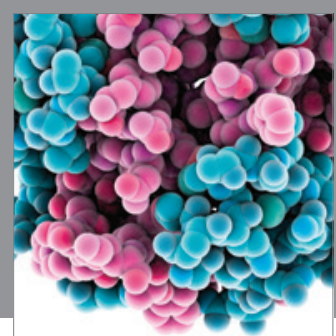

Journal of
Diabetes Research

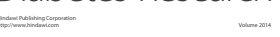

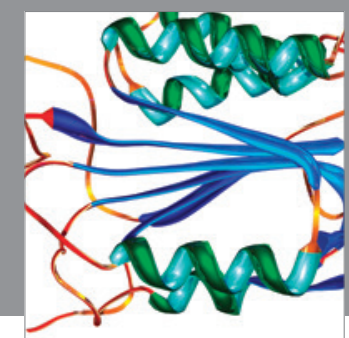

Disease Markers
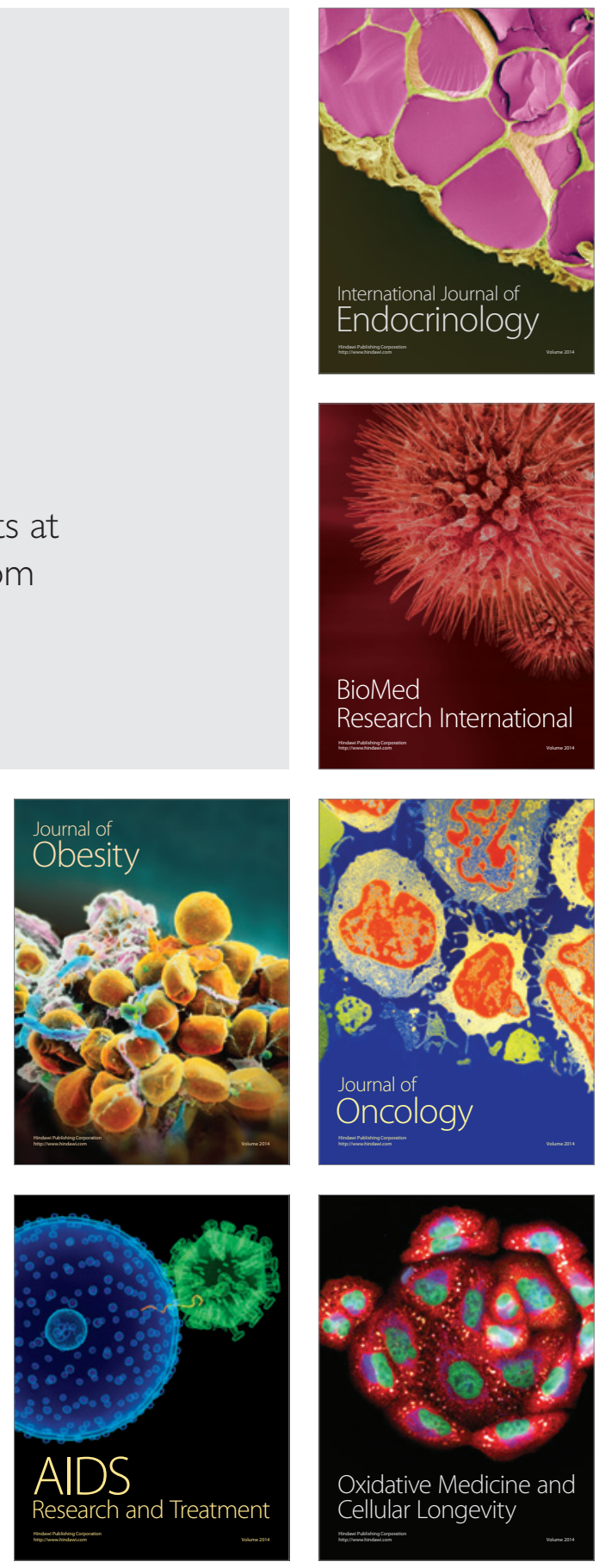